\title{
Epinephrine Autoinjector Prescription Filling after Pediatric Emergency Department Discharge
}

\author{
Joanna Cohen ${ }^{1}$, Chisom Agbim ${ }^{1}$, Michael Hrdy ${ }^{1}$, Mary Mottla ${ }^{1}$, Monika Goyal ${ }^{1}$, and \\ Kristen Breslin ${ }^{1}$ \\ ${ }^{1}$ Children's National Medical Center
}

May 27, 2020

\begin{abstract}
Background: There are known racial and socioeconomic disparities in the use of epinephrine autoinjectors for anaphylaxis. Objective: To measure rates of epinephrine autoinjector prescription filling and identify patient demographic factors associated with filling rates among patients discharged from the pediatric emergency department Methods: This was a retrospective observational cohort study of all patients discharged from a pediatric emergency department who received an outpatient prescription for an epinephrine autoinjector between January 1, 2018 and October 31, 2019. Rates of prescription filling were calculated, and multivariable logistic regression was performed to identify sociodemographic factors associated with prescription filling. Results: Of 717 patients included in the analysis, 54.8\% (95\% CI 51.1,58.5) filled their prescription. There were no significant associations between EAI fill rates and patient age or sex. In bivariable analysis, Non-Hispanic white patients were more likely to fill EAI prescriptions compared to Non-Hispanic black patients (OR 1.89, 95\% CI 1.11, 3.20) and patients with in-state Medicaid were significantly less likely to fill EAI prescriptions compared to those patients with private insurance (OR $0.69,95 \%$ CI $0.48,0.98)$. However, after multivariable adjustment there was no significant difference in filling by age, insurance status or race/ethnicity. Conclusions: Only approximately half the patients filled their EAI prescriptions after discharge. Filling rates do not vary by sociodemographic characteristics.
\end{abstract}

\section{Background and Significance}

Anaphylaxis is a rapid-onset, potentially life-threatening systemic allergic reaction. ${ }^{1,2}$ Immediate administration of intramuscular epinephrine can prevent mortality, hospitalization and biphasic reactions. ${ }^{345}$ For these reasons, national and international guidelines recommend prescribing an epinephrine autoinjector (EAI) to any patient at increased risk for anaphylaxis,,${ }^{6,7}$ and specify that all patients who have experienced an anaphylactic reaction should beprovided an action plan instructing them on how and when to administer epinephrine. ${ }^{8}$ Despite these recommendations, for a multitude of reasons, EAIs are underused in the treatment of anaphylaxis. ${ }^{9}$

Socioeconomic statuslikely influences accessibility to EAIs. Since 2007 the average wholesale price of EpiPen?, the most commonly prescribed EAI, increased by $545 \%$ to around $\$ 730$ for a 2 pack in $2016 .{ }^{10}$ Children from higher income households are more likely to be prescribed an EAI, ${ }^{11}$ and children with private insurance are more likely to receive early epinephrine administration for anaphylaxis. ${ }^{12}$ In addition to socioeconomic disparities, racial and ethnic disparities in anaphylaxis treatment also exist. Non-white children are less likely to receive early epinephrine administration. ${ }^{5}$

It is unclear if these disparitiesare attributable to prescribing practices, lack of prescription filling, lack of EAI availability when needed or lack of use when needed, despite availability. The primary aim of this study was to measurerates of EAI prescription filling and to determine if there are differences in the rate of filling 
of EAI prescriptions among patients of different socioeconomic characteristicsdischarged from the pediatric emergency department (ED) after a visit for anaphylaxis.

\section{Methods}

\section{Study design and population}

This study was a retrospective observational cohort study of patients discharged after an emergency visit with an EAI prescription from either of two affiliated pediatric EDs, an academic urban pediatric ED with an annual census of 90,000 patient visits and a satellite urban free-standing pediatric ED with an additional 30,000 annual patient visits. We included data from patients that presented over a 22 -month period from January 1,2018 to October 31, 2019. Patients were excluded if they were not prescribed an EAI or if we could not determine their prescription fill status. EAI prescription fill status could be indeterminate because the patient received a handwritten prescription, had a prescription called into the pharmacy by phone or used a pharmacy that did not participate inSureScripts?e-prescribing during the study period. The Institutional Review Board approved this study.

\section{Data Collection}

We collected patient demographic data including age, sex, race and insurance information from the electronic medical record (EMR) and determined prescription filling from aninformation technology company that works in conjunction with the EMR, SureScripts? (SureScripts, Arlington, VA., USA). SureScripts?supports e-prescriptions, the electronic transmission of prescriptions between the EDs and pharmacies. As of 2018, greater than $90 \%$ of pharmacies in the study region have the capability to receive e-prescriptions from SureScripts?.

\section{Data Analysis}

The outcome of interest wasfilling of EAI prescription after discharge from the ED. The variables of interest included the followingpatient characteristics: age, sex, race, ethnicity and insurance type. We recategorizedrace and ethnicity into a single variable:Non-Hispanic white (NH-white), Non-Hispanic black (NH-black), Hispanicand other. Insurance status was categorized as commercial or Tricare, Medicaid-instate, Medicaid-out-of-state, self-pay and other/unknown. Patient age was treated as a continuous variable. We used IBM SPSS Statistics for Windows version 26 (SPSS Inc., Chicago, Ill., USA) to perform descriptive statistics and logistic regression to measure associations between prescription fill rates and demographic factors. We performed bivariable logistic regression to assess for differences in prescription filling by patient demographics and performed multivariable logistic regression to adjust for potential confounding by all covariables. Regression models were fit to derive adjusted ORs with 95\% CIs.

\section{Results}

During the study period, there were 946 patient visits identified as having an EAI prescribed from the ED. Fivevisits were excluded because the EAI was not prescribed on ED discharge. Two of these patients were admitted to the hospital and 3 additional patients were either prescribed EAIs from the ED during a non-visit encounter or were prescribed in error and discontinued prior to ED discharge. An additional 224 visits were excluded because we could not determine if the prescriptions were filled inSureScripts?. The final analysis included 717 patients.(Figure 1)

The mean age of included patients was 8.1 years $(\mathrm{SD}=5.7$ years). Approximately half of the patients were male, $71 \%$ were NH-blackand $60 \%$ had either in-state Medicaid or out-of-state Medicaid. Fiftyfourpercent $(95 \%$ CI 51.1, 58.5) ofEAI prescriptions were filled. (Table 1)

There were no significant associations between EAI fill rates and patient age (mean difference $=0.99$ years, $(95 \%$ CI $0.96,1.01)$ or $\operatorname{sex}(\mathrm{p}=0.82)$.In the bivariate analysis, $\mathrm{NH}$-white patientswere more likely to fill EAI prescriptions when compared to NH-blackpatients (OR 1.89, 95\% CI 1.11,3.20) and patients with instate Medicaid were significantly less likely to fill EAI prescriptions when compared to those patients with 
commercial or Tricare insurance (OR 0.69, 95\% CI 0.48, 0.98). However, when adjusting for covariables there was no significant difference in filling by insurance status or race/ethnicity. (Table 2)

\section{Discussion}

In this cohort of pediatric ED visits, only approximately half of EAI prescriptions were filledfollowing ED discharge.NH-white children were more likely to have EAI prescriptions filled after an ED visit for anaphylaxis than NH-blackchildren and patients with private insurance were more likely than patients with in-state Medicaid to fill an EAI prescription. However, when adjusting for multiple variables, these differences were no longer significant.

The low EAI fill rate is this study is inconsistent with a previous study that estimated pediatric EAI fill rates closer to $90 \%$; however, this studyincluded only patients with private insurance. ${ }^{13}$ Similar to our population, previous large database adult studies that include patients with a variety of pharmacy coverage have found that approximately half of patients fill their EAI. ${ }^{14}$ It's likely that the EAI fill rate in our study was more similar to that seen in adult data because of the diversity of insurance and/or socioeconomic status in our population. While insurance type may be a specific barrier to prescription filling, it may also be a marker for lower socioeconomic status, which can confer an independent risk factor for low filling rates if for instance, pharmacies in low-income areas are less likely to carry EAIs or patients of lower socioeconomic status are less likely to have transportation to get to a pharmacy.

Identifying risk factors associated with poor rates of EAI prescription filling is imperative to create targeted strategies for improving them. The objective of this study was to identify predictors associated with EAI prescription filling. We found that while $\mathrm{NH}$-white patients had a higher fill rate compared to NHblackpatients and having in-state public insurance was associated with a lower fill rate than having private insurance, there was likely a concordance between being NH-blackand having public in-state insurance.This is consistent with the knowledge that minority children are significantly more likely to have public insurance compared to white children. ${ }^{15}$ Interestingly, patients with out-of-state Medicaid did not have the same risk of low fill rates as those patients with in-state Medicaid. This study was conducted in Washington, DC which is unique in that in-state Medicaid is restricted to District of Columbia residents and therefor encompasses an entirely urban population; whereas out-of-state Medicaid is likely to be held by patients living in the Maryland and Virginia suburbs.

In order to address low fill rates of EAI prescriptions, further studies should be aimed at assessing to what extent disparities in filling are related to patient education, medication availability and cost. In addition to targeting interventions aimed at educating patients at risk for not filling EAI prescriptions, interventions aimed at improving EAI fill rates should consider changes to the system that is serving largely minority and publicly insured patients. ${ }^{16}$ For instance, providingan EAI on discharge from the ED as an alternative to providing a prescription or partnering with local pharmacies to determine availability may be beneficial.

\section{Limitations}

This study has several important limitations. Most importantly, this was a retrospective cohort, reliant on the EMR and e-prescribing records to obtain information. This resulted in a large number of excluded patient visits for which we could not determine prescription filling. In addition, patients in whom we could not determine EAI filling were slightly more likely to have out-of-state Medicaid or self-pay insurance and slightly more likely to be Hispanic. It's possible that this patient demographic is less likely to use e-prescribing or less likely to use pharmacies participating inSureScripts?. This could create a selection bias with a trend towards a lower reported EAI fill rate as this patient demographic is somewhat more likely to fill EAI prescriptions. However, the overall number of these patients was small compared to the larger population. We were also unable to determine if patients already had EAIs at home or if they had, previously or after the ED visit, received a prescription from their primary care physician or another health care provider that they may have filled. In addition, we were only able to collect a limited amount of data from the EMR and therefore have no first-hand knowledge as to the barriers to EAI filling. Finally, the unique definition of Washington, DC as an urban district that lies outside of any state, lends itself to creating variables that might not be 
generalizable to the greater population.

In conclusion, in this cohort of pediatric patients prescribed an EAI on ED discharge from the pediatric ED, only approximately half of the patients filled their EAI prescriptions. Further studies examining predictors of EAI filling are needed to develop patient and system-oriented strategies to improve prescription filling.

1. Campbell RL, Hagan JB, Manivannan V, et al. Evaluation of national institute of allergy and infectious diseases/food allergy and anaphylaxis network criteria for the diagnosis of anaphylaxis in emergency department patients. J Allergy Clin Immunol.2012;129(3):748-752.

2. Samant SA, Campbell RL, Li JT. Anaphylaxis: diagnostic criteria and epidemiology. Allergy Asthma Proc. 2013;34(2):115-119.

3. Pumphrey RS. Lessons for management of anaphylaxis from a study of fatal reactions. Clin Exp Allergy. 2000;30(8):1144-1150.

4. Lieberman P. Biphasic anaphylactic reactions. Ann Allergy Asthma Immunol. 2005;95(3):217-226; quiz $226,258$.

5. Fleming JT, Clark S, Camargo CA, Rudders SA. Early treatment of food-induced anaphylaxis with epinephrine is associated with a lower risk of hospitalization. J Allergy Clin Immunol Pract.2015;3(1):57-62.

6. Lieberman P, Nicklas RA, Randolph C, et al. Anaphylaxis-a practice parameter update 2015. Ann Allergy Asthma Immunol.2015;115(5):341-384.

7. Simons FE, Ebisawa M, Sanchez-Borges M, et al. 2015 update of the evidence base: World Allergy Organization anaphylaxis guidelines. World Allergy Organ J. 2015;8(1):32.

8. Campbell RL, Li JT, Nicklas RA, Sadosty AT, Force MotJT, Workgroup PP. Emergency department diagnosis and treatment of anaphylaxis: a practice parameter. Ann Allergy Asthma Immunol.2014;113(6):599608.

9. Prince BT, Mikhail I, Stukus DR. Underuse of epinephrine for the treatment of anaphylaxis: missed opportunities. J Asthma Allergy.2018;11:143-151.

10. Westermann-Clark E, Pepper AN, Lockey RF. Economic considerations in the treatment of systemic allergic reactions. J Asthma Allergy.2018;11:153-158.

11. Coombs R, Simons E, Foty RG, Stieb DM, Dell SD. Socioeconomic factors and epinephrine prescription in children with peanut allergy.Paediatr Child Health. 2011;16(6):341-344.

12. Huang F, Chawla K, Järvinen KM, Nowak-Węgrzyn A. Anaphylaxis in a New York City pediatric emergency department: triggers, treatments, and outcomes. J Allergy Clin Immunol. 2012;129(1):162168.e161-163.

13. Landsman-Blumberg PB, Wei W, Douglas D, Smith DM, Clark S, Camargo CA. Concordance with recommended postdischarge care guidelines among children with food-induced anaphylaxis. $J$ Pediatr.2014;164(6):1444-1448.e1441.

14. Motosue M, Bellolio MF, Van Houten HK, Shah ND, Campbell RL. Predictors of epinephrine dispensing and allergy follow-up after emergency department visit for anaphylaxis. Ann Allergy Asthma Immunol. 2017;119(5):452-458.e451.

15. Soylu TG, Elashkar E, Aloudah F, Ahmed M, Kitsantas P. Racial/ethnic differences in health insurance adequacy and consistency among children: Evidence from the 2011/12 National Survey of Children's Health. J Public Health Res. 2018;7(1):1280.

16. Clarke AR, Goddu AP, Nocon RS, et al. Thirty years of disparities intervention research: what are we doing to close racial and ethnic gaps in health care? Med Care. 2013;51(11):1020-1026. 


\section{Hosted file}

EAI Table 1.docx available at https://authorea.com/users/326965/articles/454635-epinephrineautoinjector-prescription-filling-after-pediatric-emergency-department-discharge

\section{Hosted file}

EAI Table 2.docx available at https://authorea.com/users/326965/articles/454635-epinephrineautoinjector-prescription-filling-after-pediatric-emergency-department-discharge

\section{Hosted file}

EAI Figure 1.docx available at https://authorea.com/users/326965/articles/454635-epinephrineautoinjector-prescription-filling-after-pediatric-emergency-department-discharge 